\title{
Fluorescent protein tools for studying protein dynamics in living cells: a review
}

Richard N. Day

University of Virginia Health System

Departments of Medicine and Cell Biology

PO Box 800578

Charlottesville, Virginia 22908-0578

Fred Schaufele

University of California San Francisco

Diabetes Center

S-1230, 513 Parnassus

San Francisco, California 94143-0540

\begin{abstract}
We have witnessed remarkable advances over the past decade in the application of optical techniques to visualize the genetically encoded fluorescent proteins (FPs) in living systems. The imaging of the FPs inside living cells has become an essential tool for studies of cell biology and physiology. FPs are now available that span the visible spectrum from deep blue to deep red, providing a wide choice of genetically encoded fluorescent markers. Furthermore, some FPs have been identified that have unusual characteristics that make them useful reporters of the dynamic behaviors of proteins inside cells. These additions to the FP toolbox are now being used for some very innovative live-cell imaging applications. Here, we will highlight the characteristics and uses of a few of these exceptional probes. Many different optical methods can be combined with the FPs from marine organisms to provide quantitative measurements in living systems.

(C) 2008 Society of Photo-Optical Instrumentation Engineers. [DOI: 10.1117/1.2939093]

Paper 08137SSR received Apr. 25, 2008; revised manuscript received Apr. 28, 2008; accepted for publication Apr. 28, 2008; published online Jun. 10, 2008.
\end{abstract}

\section{Introduction}

In the first century $\mathrm{AD}$, Pliny the elder wrote of rubbing the slime from a jellyfish found in the Bay of Naples on his walking stick, saying "it seems to be on fire" and that it "will light the way like a torch." "Two thousand years later, we now can fully appreciate the importance of his observation. Efforts to identify the molecular basis for the glow of the jellyfish began with Shimomura's studies of the jellyfish Aequorea victoria in the early 1960s. His biochemical analysis resulted in the purification of the blue light-emitting photoprotein, aequorin, and also indicated the presence of an autofluorescent protein in the jellyfish extracts. ${ }^{2}$ This companion protein to aequorin, the jellyfish green fluorescent protein (GFP), was later isolated by Shimomura and colleagues, who showed that GFP absorbed the blue light emission from aequorin and then reemitted it as green light. ${ }^{3}$

It is now well appreciated that many marine organisms produce fluorescent proteins (FPs) that are homologous to the Aequorea GFP. ${ }^{4}$ In reef corals, much of the color diversity results from FPs, which may function in photoprotective mechanisms, ${ }^{5}$ or might have evolved to support symbiotic relationships between the corals and algae. ${ }^{6}$ However, it is their application to a wide variety of biological systems that sparked a revolution in studies of cell biology and physiology. The revolution began with the cloning of the Aequorea GFP by Prasher ${ }^{7}$ in 1992, providing for the first time the means to express a genetically encoded fluorescence marker inside living cells and organisms. ${ }^{8,9}$ Since then, the utility of the FPs as noninvasive probes has been repeatedly proven by their integration into living systems. ${ }^{10}$

Address all correspondence to Richard N. Day, Departments of Medicine and Cell Biology, Univ. of Virginia, Jefferson Park Avenue, Charlottesville, Virginia 22908; Tel.: 434-982-3623; Fax: 434-982-0088; E-mail: rnd2v@virginia.edu

\section{Choosing a Fluorescent Protein}

We now have FPs that span the visible spectrum from deep blue to deep red, providing a wide choice of genetically encoded markers for studies in cell biology. These proteins are being used to monitor intracellular activities, such as $\mathrm{pH}$ in organelles, or the transcriptional activity of reporter genes. ${ }^{11}$ Some FPs have been identified that have unusual characteristics that make them useful reporters of the dynamic behaviors of the proteins to which they are attached. In the sections below, we will highlight a few of these exceptional probes, but our intent here is not to provide a comprehensive list of the FPs that are currently available. Shaner and colleagues ${ }^{12}$ recently published an extensive review that describes the characteristics of many important members of the FP toolbox. Most of the FPs that are used today have been modified through mutagenesis to optimize their expression in biological systems. Still, there are several important considerations in choosing the best FP for a particular application.

Many of the FPs that have been characterized from marine organisms are either dimers, tetramers, or part of higher-order complexes. ${ }^{13}$ In this regard, although the Aequorea GFP could be crystallized as a monomer, it will form dimers when the protein is highly concentrated. ${ }^{14-16}$ The dimerization is mediated by hydrophobic amino acids in the carboxyl-terminus of the Aequorea-based FPs. ${ }^{16}$ Although dimerization is not typically observed when the FPs are free to diffuse within the cell, there is a tendency to form dimers when the proteins are expressed in a restricted volume, such as in the two-dimensional space of biological membranes. Here, the dimerization of the FPs can cause atypical complexes to form between the proteins that they are linked to. ${ }^{17}$ To overcome this problem, Zacharias and colleagues ${ }^{16}$ developed monomeric forms of

1083-3668/2008/13(3)/031202/6/\$25.00 (C) 2008 SPIE 
the Aequorea-based FPs by substitution of the alanine at position 206 with lysine, blocking the dimer formation without altering the fluorescence characteristics.

When cellular proteins are labeled with the FPs, it is critical that the $27 \mathrm{kDa}$ tag does not interfere with the normal cellular functions of the expressed protein. Here, rigorous control experiments are necessary to verify that the fusion proteins behave like their endogenous counterparts. In addition, it must be recognized that any amount of fusion protein expressed in cells is, by definition, over-expression relative to the endogenous protein. While transient transfection provides the greatest flexibility for analyzing protein function, this approach can yield very high levels of fusion-protein expression in the target cells. This can result in improper subcellular distribution and protein dysfunction that could lead to erroneous interpretations of protein localization and activity.

When selecting among the many color variants that are now available, it is important to consider that live-cell imaging is a tradeoff between acquiring adequate signal from the expressed FPs, while limiting cell damage that might be caused by the illumination of the fluorophores. Living systems are more tolerant of longer wavelength illumination than of light near the ultraviolet (UV) wavelengths. ${ }^{18}$ Further, fluorophores in the excited state can produce highly reactive free radicals that are potentially damaging to cells, so it is important to minimize the exposure of the living cells to the excitation illumination. ${ }^{19}$ Selecting bright FPs that are excited at longer wavelengths will help minimize the potential for photodamage. If a blue FP is needed, however, long wavelength excitation can be achieved with two-photon excitation microscopy, offering a less damaging alternative to near UV excitation. ${ }^{18,20}$ Finally, these potential problems can also be mitigated by improving the light gathering capabilities of the microscope by using high-numerical-aperture objectives, and by increasing the sensitivity of the detector.

\section{The Fluorescent Protein Color Palette}

The current FP color palette includes modified proteins based on the Aequorea GFP, as well as a variety of FPs that were cloned from other marine organisms and improved for livecell imaging applications through genetic engineering. The sequence encoding the Aequorea GFP was engineered over the years to yield new FPs emitting light in the blue to yellowish green range of the spectrum. ${ }^{11,21}$ These optimized blue, ${ }^{22}$ cyan, ${ }^{23}$ green, ${ }^{24}$ and yellow ${ }^{25}$ FPs (see Table 1) expanded the repertoire of uses to include multicolor imaging of protein co-localization (see Fig. 1) and, importantly, their applications for Förster resonance energy transfer (FRET) microscopy to measure protein-protein interactions (discussed below). Furthermore, many GFP-like proteins that are responsible for the bright colors that we see in reef corals have been characterized, cloned, and optimized for imaging applications. ${ }^{12,26}$ For instance, the mushroom anemone Discosoma striata provided us with the first of the red FPs (RFP), called DsRed. ${ }^{27}$

There were problems, however, with DsRed as a probe in living cells, and mutagenesis strategies were used to markedly improve the FP. ${ }^{28-30}$ This approach yielded a rapid maturing monomeric RFP (mRFP), but even this new FP had limited usefulness as a probe for quantitative imaging studies. ${ }^{30}$ To generate new FPs with improved characteristics, Tsien's laboratory subjected mRFP to many rounds of directed evolution using both the error prone polymerase chain reaction and somatic hypermutation in B-lymphocytes. ${ }^{31,32}$ When combined with cell-based screening methods, these approaches yielded an entirely new variety of FPs. This new crop of FPs included mCherry, which is a rapid maturing, bright, stable version of mRFP (Table 1). In addition, a dimeric FP called tdTomato was generated that is currently the brightest of the available FPs (Table 1). This probe is useful for applications that require minimal exposure to excitation illumination to maintain cell viability.

Still other novel FPs have been cloned from corals and engineered to improve their utility for live-cell imaging. For example, Campbell's laboratory used the directed evolution of a sequence encoding a cyan-colored protein from the coral Clavularia to generate a monomeric teal fluorescent protein (mTFP) with remarkable brightness and photostability (Table 1). ${ }^{33}$ The mTFP is noteworthy because it filled the spectral gap between the cyan and green FPs, and it is optimally excited by the 458-nm laser line that is available on most confocal microscopes. Another notable FP is a yellow-orange protein that was derived from mushroom coral Fungia concinna. This FP was engineered to a bright, photostable monomeric protein called Kusabira orange (mKO; see Table 1). ${ }^{34}$ Another FP that warrants mention is a deep red protein called Katushka. This FP was engineered to the monomeric protein known as mKate, which is currently the brightest and most photostable of the deep red FPs, making it potentially useful for imaging in intact animals. ${ }^{35}$

These additions to the FP toolbox are being used for some very innovative live-cell imaging applications. For example, Lichtman's laboratory ${ }^{36}$ recently combined the sequences encoding several different FPs into common reporter cassettes, and then used these to generate transgenic mice. Here, lox recombination sites were inserted between the sequences encoding the different FPs. This arrangement permitted Cremediated recombination to randomly determine which of the FPs would be expressed from a particular cassette. Remarkably, when multiple copies of the cassette were targeted to the mouse neurons, the many different random color combinations generated allowed individual neurons within entire neural networks to be distinguished from their neighbors. ${ }^{36}$ This system, called Brainbow, is allowing the investigators to determine how cellular connections are established in neural circuits, information that will be critical for understanding how the nervous system works.

\section{The Photoactivatable FPs}

Some of the naturally occurring FPs display a characteristic called "kindling," where they are initially weakly fluorescent, but can then become brightly fluorescent in response to intense illumination. ${ }^{37,38}$ This quality can be extremely useful since, unlike the FPs that are uniformly fluorescent from the time they are produced, the photoactivatable FPs can be "switched on" at a particular time and location within the cell to track the behavior of a tagged protein. Very weak kindling behavior was observed for the wild-type Aequorea GFP following intense illumination with near UV light. The Aequorea GFP is optimally excited at $395 \mathrm{~nm}$, but also has a minor 
Day and Schaufele: Fluorescent protein tools for studying protein dynamics...

Table 1 Selected tools from the fluorescent protein toolbox.

\begin{tabular}{|c|c|c|c|c|c|c|}
\hline FP & $\begin{array}{l}\text { Peak Ex } \\
\qquad(\mathrm{nm})\end{array}$ & $\begin{array}{l}\text { Peak Em } \\
(\mathrm{nm})\end{array}$ & $\begin{array}{c}\text { Intrinsic } \\
\text { Brightness }\end{array}$ & Photostability & Distinction & Ref. \\
\hline \multicolumn{7}{|c|}{ Aequorea-based color variants } \\
\hline EBFP2 & 383 & 448 & 18 & ++ & Brightest blue & 22 \\
\hline Cerulean & $433-445$ & $475-503$ & 27 & ++ & $\begin{array}{l}\text { Bright cyan, proven } \\
\text { FRET donor }\end{array}$ & 23 \\
\hline Emerald & 487 & 509 & 39 & ++++ & Bright, stable green & 24 \\
\hline Venus & 515 & 528 & 53 & $++^{b}$ & $\begin{array}{l}\text { Bright yellowish, proven } \\
\text { FRET acceptor }\end{array}$ & 25 \\
\hline \multicolumn{7}{|c|}{ mRFP-based color variants } \\
\hline tdTomato & 554 & 581 & 95 & +++ & $\begin{array}{l}\text { Brightest FP, but } \\
\text { dimeric }\end{array}$ & 32 \\
\hline mCherry & 587 & 610 & $17^{\mathrm{c}}$ & +++ & Rapid maturing red & 32 \\
\hline \multicolumn{7}{|c|}{ Color variants from corals } \\
\hline mTFP & 462 & 492 & 54 & +++ & Bright teal, FRET donor & 33 \\
\hline $\begin{array}{l}\text { Kusabira } \\
\text { Orange }\end{array}$ & 548 & 559 & 31 & +++ & Bright orange & 34 \\
\hline mKate & 588 & 635 & 15 & ++++ & Most stable deep red & 35 \\
\hline \multicolumn{7}{|c|}{ Photoconvertible variants } \\
\hline $\begin{array}{l}\text { PA-GFP } \\
\text { (before) }^{d}\end{array}$ & 400 & 515 & 3 & $\begin{array}{l}\text { Weak Ex } \\
\text { at } 488 \mathrm{~nm}\end{array}$ & $\begin{array}{l}\text { Fluorescent marker; blue light } \\
\text { induced photoactivation }\end{array}$ & 40 \\
\hline $\begin{array}{l}\text { PA-GFP } \\
\left(_{(\text {after })^{\mathrm{e}}}\right.\end{array}$ & 504 & 517 & 14 & $\begin{array}{l}\text { Stable } \\
\text { green }\end{array}$ & & \\
\hline $\begin{array}{l}\text { Dendra } \\
{\text { (before })^{d}}^{\text {(bef }}\end{array}$ & 490 & 507 & 23 & $\begin{array}{l}\text { Stable } \\
\text { green }\end{array}$ & $\begin{array}{l}\text { Fluorescent highlighter; blue light induced } \\
\text { green to red conversion }\end{array}$ & 45 \\
\hline $\begin{array}{l}\text { Dendra } \\
\text { (after) }^{\mathrm{e}}\end{array}$ & 553 & 573 & 19 & $\begin{array}{l}\text { Stable } \\
\text { red }\end{array}$ & & \\
\hline $\begin{array}{l}\text { Dronpa } \\
\text { (before) }\end{array}$ & 503 & 518 & 80 & $\begin{array}{l}\text { Unstable } \\
\text { green }\end{array}$ & $\begin{array}{l}\text { Sequential marker; repeated switching } \\
\text { from dark to fluorescent state }\end{array}$ & 42 \\
\hline
\end{tabular}

antrinsic brightness is the product of quantum yield and extinction coefficient.

broven useful for applications that involve photobleaching.

${ }^{c}$ Cellular autofluorescence is low at longer wavelengths, improving the signal-to-noise ratio.

dBefore photoactivation or photoconversion.

${ }^{\mathrm{e}}$ After photoactivation or photoconversion.

absorbance peak near $480 \mathrm{~nm}$. Following brief intense illumination with near UV light, there is a change in the protonation state of the chromophore, resulting in an enhancement of the absorbance near $480 \mathrm{~nm} .{ }^{39}$ Mutations were introduced into the Aequorea GFP that greatly increased its kindling behavior, resulting in a strongly photoactivatable GFP (PA-GFP). ${ }^{40}$ Now, when PA-GFP is exposed to a brief pulse of intense 400-nm light, there is a 100-fold increase in its excitation at $488 \mathrm{~nm}$ (Table 1). ${ }^{40}$ When PA-GFP-labeled proteins are photoactivated inside the living cell, the diffusion of the newly fluorescent proteins provides a direct measure of the mobility of the labeled proteins. ${ }^{41}$
Another FP that displays a more extreme type of kindling behavior was recently identified in the Pectinia $s p$. coral. ${ }^{42}$ This protein, called Dronpa, was isolated as part of an oligomeric complex and engineered to a monomeric form that emits bright green fluorescence when illuminated at $490 \mathrm{~nm}$. The fluorescence signal from Dronpa, however, is rapidly lost under continued illumination as the protein is driven into a reversible dark state. Significantly, brief exposure to intense 405-nm light can be used to "switch on" the bright green fluorescence back on. This light-activated generation of different protonation states in the chromophore permits Dronpa to be repeatedly switched between the fluorescent and the 

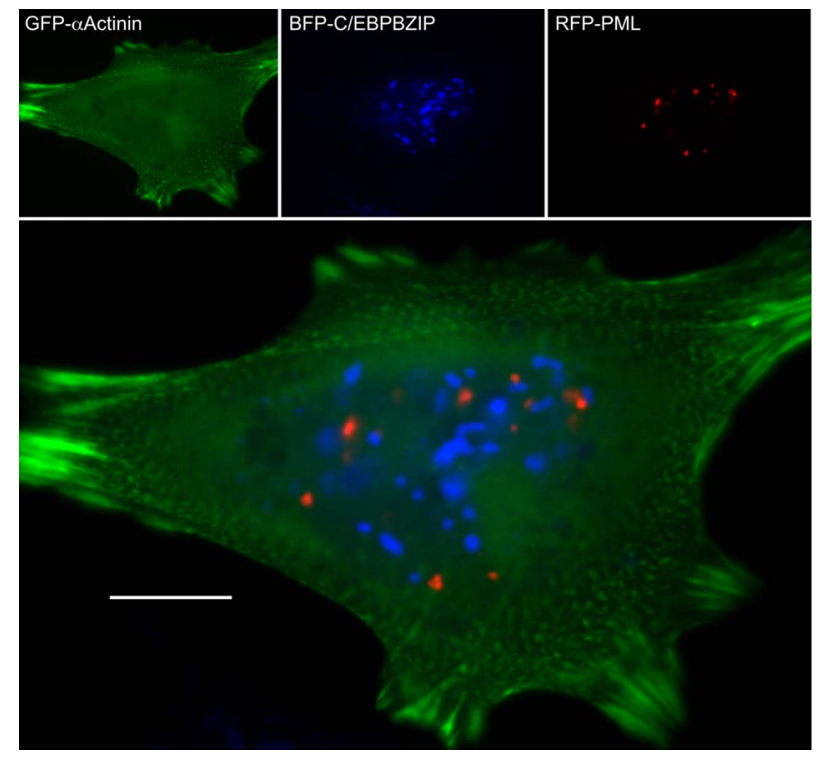

Fig. 1 Multichannel imaging to visualize the subcellular localization of proteins tagged with spectrally distinct FPs. Mouse pituitary GHFT1 cells were transfected with a mixture of plasmid DNAs that encoded for the expression of GFP- $\alpha$ Actinin (cytoplasmic, cytoskeleton), BFP-C/EBP $\alpha$ basic-leucine zipper domain (BZIP; nucleus, heterochromatin domains), and RFP-promyelocytic leukemia protein (PML; nucleus, nuclear bodies). The upper panels show the individual images acquired in each of the three channels, while the lower panel shows the merged, three-color image (the calibration bar is $10 \mu \mathrm{m}$ ). (Color online only.)

dark states (Table 1 ). ${ }^{42,43}$ The ability to sequentially highlight and then erase the fluorescence from Dronpa was recently used to analyze the anatomy of neural networks in developing Zebra fish embryos. ${ }^{44}$ In addition to photoswitching, still other new FPs have been identified that can change their color when illuminated at specific wavelengths of light. For example, a photoconvertable FP called Dendra was isolated from the stony coral Trachyphyllia. Dendra emits green light, but when illuminated with near UV light, the protein is stably converted to an RFP (Table 1). ${ }^{45}$ These FPs with unique photoswitching and photoconversion capabilities are providing new ways to measure the dynamic behaviors of proteins inside living cells. $^{46}$

\section{Quantifying Protein Behavior}

A variety of optical methods can exploit the characteristics of the FPs. For example, fluorescence correlation spectroscopy (FCS) can be used to directly measure protein dynamics in living cells. The FCS approach uses the microscope objective lens to focus the laser beam into the specimen, creating a diffraction-limited excitation volume. FCS is essentially a photon-counting technique that monitors the fluorescence signal emanating from the very small $(\sim 1 \mathrm{fL})$ optically defined volume. The fluorescence signal coming from the observation volume fluctuates as the labeled molecules diffuse in and out, and the duration of the fluctuations are related to the average time individual molecules reside within volume. This residence time can be used to determine the diffusion coefficient for the FP-labeled proteins in the volume. ${ }^{47}$
The spectral properties of the FPs allow them to be used in Förster (fluorescence) resonance energy transfer (FRET) microscopy to measure the spatial relationships between proteins. FRET microscopy measures the effect of the direct transfer of excited-state energy from a donor FP to nearby acceptor FPs. This energy transfer results from an electronic coupling between the donor and acceptor fluorophores, and this limits the distance over which energy transfer can occur between the FPs to less than about $80 \AA$. This allows the detection of FRET to provide unique information about the spatial relationships between proteins in living cells on the scale of angstroms. ${ }^{48,49}$ A critical requirement for FRET is a strong overlap between the donor emission spectrum and the absorption spectrum of the acceptor. In this regard, increasing the spectral overlap between the donor and acceptor FPs strengthens their electronic coupling. However, factors beyond spectral overlap also appear to play a role in determining whether a particular pair of FPs will be useful for FRET measurements. We, and others (M. Davidson, Florida State University, personal communication, 2008) have observed that, despite having optimal spectral overlaps, not all FPs behave as expected for FRET applications. Thus, it is important not only to consider the spectral characteristics, but to also test new FP pairings for their efficacy in FRET-based assays. Currently, the FPs based on cyan fluorescent protein (CFP) and yellow fluorescent protein (YFP) remain the most popular FRET pair. ${ }^{50}$ The new mTFP (see Table 1) generated in Campbell's laboratory ${ }^{33}$ is also a useful donor FP for FRET measurements from living cells. ${ }^{51}$ Recently, Campbell's group also reported the development of a new FP with an exceptionally large Stoke's shift called Ametrine that appears well suited for FRET applications. ${ }^{52}$

Energy transfer can also be detected by measuring the fluorescence lifetime of a fluorophore, the time the fluorophore spends in the excited state before returning to the ground state. ${ }^{49}$ Fluorescence lifetime imaging microscopy (FLIM) techniques can be used to measure the lifetimes of fluorophores in living cells. Where conventional microscopy might detect similar fluorescence intensities, FLIM can detect regional differences in the fluorescence lifetimes. Since the fluorescence lifetime of a fluorophore is sensitive to any process that influences the excited state, energy transfer can be measured by FLIM. When FRET occurs, the presence of the acceptors will cause a shift in the mean lifetime for the donor population to shorter lifetimes. These FLIM-FRET measurements can potentially provide very detailed information about molecular interactions in living cells.

\section{Conclusion}

Over the past decade, the remarkable advances associated with the fluorescent proteins and live-cell imaging approaches have made these essential tools for studies of cell biology and physiology. Just as with any approach, however, the control experiments for the FP-based studies are very important. Further, these live-cell imaging approaches, by themselves, do not prove the critical functions of proteins. Rather, these noninvasive techniques serve to complement and extend the results that are obtained by the biochemical analysis of the endogenous cellular proteins. Despite these limits, the measurements of the FPs in the natural environment inside 
living cells provide the most physiologically relevant information currently available. Just as Pliny observed, it is fair to say that the fluorescent proteins will continue to light the way.

\section{Acknowledgments}

We thank Michael Davidson, Robert Campbell, Carol Otey, George Patterson, Atsushi Miyawaki, David Piston, and Roger Tsien for their generous contribution of FPs. We also thank Mike Davidson for his contribution of technical information about new FPs, and we thank Cynthia Booker and Kathy Day for their critical reading of this manuscript.

\section{References}

1. Pliny, Natural History, Vol. 8, Books $28-32$, p. 551, trans. W.H.S Jones, Loeb Classical Library, Harvard University Press, Cambridge, MA (1963).

2. O. Shimomura, F. H. Johnson, and Y. Saiga, "Extraction purification and properties of aequorin, a bioluminescent protein from the luminous hydromedusan, Aequorea," J. Cell. Comp. Physiol. 59, 223-239 (1962).

3. H. Morise, O. Shimomura, F. H. Johnson, and J. Winant, "Intermolecular energy transfer in the bioluminescent system of Aequorea," Biochemistry 13, 2656-2662 (1974).

4. M. V. Matz, K. A. Lukyanov, and S. A. Lukyanov, "Family of the green fluorescent protein: journey to the end of the rainbow," BioEssays 24, 953-959 (2002).

5. A. Salih, A. Larkum, G. Cox, M. Kühl, and O. Hoegh-Guldberg, "Fluorescent pigments in corals are photoprotective," Nature (London) 408, 850-853 (2000).

6. S. F. Field, M. Y. Bulina, I. V. Kelmanson, J. P. Bielawski, and M. V. Matz, "Adaptive evolution of multicolored fluorescent proteins in reef-building corals," J. Mol. Evol. 62, 332-339 (2006).

7. D. C. Prasher, V. K. Eckenrode, W. W. Ward, F. G. Prendergast, and M. J. Cormier, "Primary structure of the Aequorea victoria greenfluorescent protein," Gene 111, 229-233 (1992).

8. M. Chalfie, Y. Tu, G. Euskirchen, W. W. Ward, and D. C. Prasher, "Green fluorescent protein as a marker for gene expression," Science 263, 802-805 (1994).

9. S. Inouye and F. I. Tsuji, "Aequorea green fluorescent protein, expression of the gene and fluorescence characteristics of the recombinant protein," FEBS Lett. 341, 277-280 (1994).

10. A. K. Hadjantonakis, M. E. Dickinson, S. E. Fraser, and V. E. Papaioannou, "Technicolour transgenics: imaging tools for functional genomics in the mouse," Nat. Rev. Genet. 4, 613-625 (2003).

11. R. Y. Tsien, "The green fluorescent protein," Аппи. Rev. Biochem. 67, 509-544 (1998).

12. N. C. Shaner, G. H. Patterson, and M. W. Davidson, "Advances in fluorescent protein technology," J. Cell. Sci. 120, 4247-4260 (2007)

13. D. A. Shagin, E. V. Barsova, Y. G. Yanushevich, A. F. Fradkov, K. A. Lukyanov, Y. A. Labas, T. N. Semenova, J. A. Ugalde, A. Meyers, J. M. Nunez, E. A. Widder, S. A. Lukyanov, and M. V. Matz, "GFP-like proteins as ubiquitous metazoan superfamily: evolution of functional features and structural complexity," Mol. Biol. Evol. 21, 841-850 (2004).

14. M. Ormö, A. B. Cubitt, K. Kallio, L. A. Gross, R. Y. Tsien, and S. J. Remington, "Crystal structure of the Aequorea victoria green fluorescent protein," Science 273, 1392-1395 (1996).

15. K. Brejc, T. K. Sixma, P. A. Kitts, S. R. Kain, R. Y. Tsien, M. Ormo, and S. J. Remington, "Structural basis for dual excitation and photoisomerization of the Aequorea victoria green fluorescent protein," Proc. Natl. Acad. Sci. U.S.A. 94, 2306-2311 (1997).

16. D. A. Zacharias, J. D. Violin, A. C. Newton, and R. Y. Tsien, "Partitioning of lipid-modified monomeric GFPs into membrane microdomains of live cells," Science 296, 913-916 (2002).

17. A. Kenworthy, "Peering inside lipid rafts and caveolae," Trends Biochem. Sci. 27, 435-437 (2002)

18. J. M. Squirrell, D. L. Wokosin, J. G. White, and B. D. Bavister, "Long-term two-photon fluorescence imaging of mammalian embryos without compromising viability," Nat. Biotechnol. 17, 763-767 (1999).

19. D. J. Stephens and V. J. Allan, "Light microscopy techniques for live cell imaging," Science 300, 82-86 (2003).
20. H. Wallrabe, M. Stanley, A. Periasamy, and M. Barroso, "One- and two-photon fluorescence resonance energy transfer microscopy to establish a clustered distribution of receptor-ligand complexes in endocytic membranes," J. Biomed. Opt. 8, 339-346 (2003).

21. J. Zhang, R. E. Campbell, A. Y. Ting, and R. Y. Tsien, "Creating new fluorescent probes for cell biology," Nat. Rev. Mol. Cell Biol. 3, 906918 (2002)

22. H. W. Ai, N. C. Shaner, Z. Cheng, R. Y. Tsien, and R. E. Campbell, "Exploration of new chromophore structures leads to the identification of improved blue fluorescent proteins," Biochemistry 46, 59045910 (2007).

23. M. A. Rizzo, G. H. Springer, B. Granada, and D. W. Piston, "An improved cyan fluorescent protein variant useful for FRET," Nat. Biotechnol. 22, 445-449 (2004).

24. A. B. Cubitt, L. A. Woollenweber, and R. Heim, "Understanding structure-function relationships in the Aequorea victoria green fluorescent protein," Methods Cell Biol. 58, 19-30 (1999).

25. T. Nagai, K. Ibata, E. S. Park, M. Kubota, K. Mikoshiba, and A Miyawaki, "A variant of yellow fluorescent protein with fast and efficient maturation for cell-biological applications," Nat. Biotechnol. 20, 87-90 (2002).

26. Y. A. Labas, N. G. Gurskaya, Y. G. Yanushevich, A. F. Fradkov, K. A. Lukyanov, S. A. Lukyanov, and M. V. Matz, "Diversity and evolution of the green fluorescent protein family," Proc. Natl. Acad. Sci. U.S.A. 99(7), 4256-4261 (2002).

27. M. V. Matz, A. F. Fradkov, Y. A. Labas, A. P. Savitsky, A. G Zaraisky, M. L. Markelov, and S. A. Lukyanov, "Fluorescent proteins from nonbioluminescent Anthozoa species," Nat. Biotechnol. 17, 969-973 (1999).

28. G. S. Baird, D. A. Zacharias, and R. Y. Tsien, "Biochemistry, mutagenesis, and oligomerization of DsRed, a red fluorescent protein from coral," Proc. Natl. Acad. Sci. U.S.A. 97, 11984-11989 (2000).

29. B. J. Bevis and B. S. Glick, "Rapidly maturing variants of the Discosoma red fluorescent protein (DsRed)," Nat. Biotechnol. 20, 83-87 (2002).

30. R. E. Campbell, O. Tour, A. E. Palmer, P. A. Steinbach, G. S. Baird, D. A. Zacharias, and R. Y. Tsien, "A monomeric red fluorescent protein," Proc. Natl. Acad. Sci. U.S.A. 99, 7877-7882 (2002).

31. L. Wang, W. C. Jackson, P. A. Steinbach, and R. Y. Tsien, "Evolution of new nonantibody proteins via iterative somatic hypermutation," Proc. Natl. Acad. Sci. U.S.A. 101, 16745-16749 (2004).

32. N. C. Shaner, R. E. Campbell, P. A. Steinbach, B. N. Giepmans, A. E. Palmer, and R. Y. Tsien, "Improved monomeric red, orange and yellow fluorescent proteins derived from Discosoma sp. red fluorescent protein," Nat. Biotechnol. 22, 1567-1572 (2004).

33. H. W. Ai, N. J. Henderson, S. J. Remington, and R. E. Campbell, "Directed evolution of a monomeric bright and photostable version of Clavularia cyan fluorescent protein: structural characterization and applications in fluorescence imaging," Biochem. J. 400, 531-540 (2006).

34. S. Karasawa, T. Araki, T. Nagai, H. Mizuno, and A. Miyawaki, "Cyan-emitting and orange-emitting fluorescent proteins as a donor/ acceptor pair for fluorescence resonance energy transfer," Biochem. J. 381, 307-312 (2004).

35. D. Shcherbo, E. M. Merzlyak, T. V. Chepurnykh, A. F. Fradkov, G. V Ermakova, E. A. Solovieva, K. A. Lukyanov, E. A. Bogdanova, A. G. Zaraisky, S. Lukyanov, and D. M. Chudakov, "Bright far-red fluorescent protein for whole-body imaging," Nat. Methods 4, 741-746 (2007).

36. J. Livet, T. A. Weissman, H. Kang, R. W. Draft, J. Lu, R. A. Bennis, J. R. Sanes, and J. W. Lichtman, "Transgenic strategies for combinatorial expression of fluorescent proteins in the nervous system," Nature (London) 450, 56-62 (2007).

37. D. M. Chudakov, V. V. Belousov, A. G. Zaraisky, V. V. Novoselov, D. B. Staroverov, D. B. Zorov, S. Lukyanov, and K. A. Lukyanov, "Kindling fluorescent proteins for precise in vivo photolabeling," Nat. Biotechnol. 21, 191-194 (2003).

38. D. M. Chudakov, A. V. Feofanov, N. N. Mudrik, S. Lukyanov, and K. A. Lukyanov, "Chromophore environment provides clue to "kindling fluorescent protein" riddle," J. Biol. Chem. 278, 7215-7219 (2003).

39. H. Yokoe and T. Meyer, "Spatial dynamics of GFP-tagged proteins investigated by local fluorescence enhancement," Nat. Biotechnol. 14, 1252-1256 (1996)

40. G. H. Patterson and J. Lippincott-Schwartz, "A photoactivatable GFP 
for selective photolabeling of proteins and cells," Science 297, 18731877 (2002).

41. J. Lippincott-Schwartz, N. Altan-Bonnet, and G. H. Patterson, "Photobleaching and photoactivation: following protein dynamics in living cells," Nat. Cell Biol. 5(Suppl), S7-S14 (2003).

42. R. Ando, H. Mizuno, and A. Miyawaki, "Regulated fast nucleocytoplasmic shuttling observed by reversible protein highlighting," Science 306, 1370-1373 (2004).

43. M. Andresen, A. C. Stiel, S. Trowitzsch, G. Weber, C. Eggeling, W. C. Wahl, S. W. Hell, and S. Jakobs, "Structural basis for reversible photoswitching in Dronpa," Proc. Natl. Acad. Sci. U.S.A. 104, 13005-13009 (2007).

44. S. Aramaki and K. Hatta, "Visualizing neurons one-by-one in vivo: optical dissection and reconstruction of neural networks with reversible fluorescent proteins," Dev. Dyn. 235, 2192-2199 (2006).

45. N. G. Gurskaya, V. V. Verkhusha, A. S. Shcheglov, D. B. Staroverov, T. V. Chepurnykh, A. F. Fradkov, S. Lukyanov, and K. A. Lukyanov, "Engineering of a monomeric green-to-red photoactivatable fluorescent protein induced by blue light," Nat. Biotechnol. 24, 461-465 (2006).
46. K. A. Lukyanov, D. M. Chudakov, S. Lukyanov, and V. V. Verkhusha, "Innovation: Photoactivatable fluorescent proteins," Nat. Rev. Mol. Cell Biol. 6, 885-891 (2005).

47. K. M. Berland, "Fluorescence correlation spectroscopy: a new tool for quantification of molecular interactions," Methods Mol. Biol. 261 , 383-398 (2004).

48. E. A. Jares-Erijman and T. M. Jovin, "FRET imaging," Nat. Biotechnol. 21, 1387-1395 (2003).

49. A. Periasamy and R. N. Day, Molecular Imaging: FRET Microscopy and Spectroscopy, Oxford University Press, New York (2005).

50. S. V. Koushik, H. Chen, C. Thaler, H. L. Puhl III, and S. S. Vogel, "Cerulean, Venus, and VenusY67C FRET reference standards," Biophys. J. 91(12), L99-L101 (2006).

51. R. N. Day, C. F. Booker, and A. Periasamy, "Characterization of an improved donor fluorescent protein for Förster resonance energy transfer microscopy," J. Biomed. Opt. 13, 031203 (2008).

52. H. W. Ai, K. L. Hazelwood, M. W. Davidson, and R. E. Campbell, "Fluorescent protein FRET pairs for ratiometric imaging of dual biosensors," Nat. Methods 5, 401-403 (2008). 